\title{
DERRUMBE PARCIAL DEL TECHO DE UNA SALA DEL MUSEO JESUÍTICO NACIONAL DE JESÚS MARÍA. ANÁLISIS ESTRUCTURAL Y PROYECTO DE RECUPERACIÓN
}

\author{
J. L. GÓMEZ, M. DEL C. FERNÁNDEZ SAIZ, K. KLEIN \\ Taller de Investigación de Diseño Estructural, Facultad de Arquitectura, Urbanismo y Diseño, \\ Universidad Nacional de Córdoba, Córdoba, Argentina.
}

\begin{abstract}
RESUMEN
En este trabajo se analizan las posibles causas que han producido el derrumbe parcial del techo de una sala del Museo Jesuítico Nacional de Jesús María, declarado Patrimonio de la Humanidad. Como antecedente se cuenta con la verificación realizada en ese sector en el año 2008 por un grupo de investigación de la UNC al cual pertenecían los autores de este trabajo. En esa verificación se habían considerado las dimensiones externas de los elementos de madera como si las secciones fueran macizas. Cuando se enviaron muestras para la identificación del material a la Facultad de Ingeniería Forestal de la U.N.S.E. se informó que la madera era algarrobo y que habían detectado galerías producidas por insectos en las muestras. Por ese motivo en las Recomendaciones de Actuación se indicó la desinfección de toda la estructura de madera del Museo, pues estos canales disminuyen las características mecánicas de las mismas. Ocurrido el derrumbe, y después de haber sido clasificadas las diferentes piezas, se extrajo una muestra del par de la cabriada afectada obteniendo una probeta de $5 \times 5 \times 15 \mathrm{~cm}$, como prescribe la norma NBR 7190 para realizar ensayo a compresión. También se pudo ensayar a flexión una correa perteneciente al mismo techo. Aunque los resultados de estos ensayos indican una resistencia menor que las obtenidas con maderas sin defectos, se aconsejó recuperar los elementos dañados utilizando la madera original, realizando refuerzos y uniones con varillas roscadas zincadas y resinas epoxi en cada situación, de acuerdo a bibliografía especializada en recuperación de estructuras de maderas en edificios patrimoniales.
\end{abstract}

Palabras clave: Patrimonio; Verificación Estructural; Recuperación; Preservación

\begin{abstract}
In this document there are analyzed the possible causes for the partial collapse of the roof of a room at the National Jesuitical Museum of Jesus Maria, declared World Heritage Site. As precedent, there is the verification conducted in this area in 2008 by a research group from UNC to which were part of the authors of this work. In this verification had been considered the external dimensions of the wood elements as if the sections were solid. When the samples were sent for identification of the material to the Forest Engineering Faculty of the UNSE it was reported that the wood was carob and that there had been detected galleries produced by insects in the samples. For this reason, it was indicated in the Action Recommendations to disinfect the wood structure of the museum because these galleries diminish the mechanical characteristics of it. After the collapse and after the classification of the different pieces, it was extracted a sample of the pair of the truss affected, obtaining a sample of $5 \times 5 \times 15 \mathrm{~cm}$ following the procedures established by the NBR 7190 regulation. Also, a purlin from the same roof was tested. Although the results from these tests indicate a lower resistance than the obtained from the wood without the defects, it was recommended to recover the damaged elements utilizing the original wood, performing reinforces and bonds with zinc threaded bars and epoxy in every situation, according to bibliography specialized in the recovery of wood structures in patrimonial buildings.
\end{abstract}

Key words: Heritage, Structural Verification, Recovery, Preservation 


\section{INTRODUCCIÓN}

El Museo Jesuítico San Isidro Labrador (1618), se encuentra en la ciudad de Jesús María, que está ubicada en el centro-norte de la provincia de Córdoba (Argentina), a $50 \mathrm{~km}$ de la Capital provincial, con la que se vincula por la Ruta Nacional $N^{\circ} 9$.

El conjunto incluye además del Museo, la iglesia, la residencia y la bodega, los restos de los antiguos molinos, el perchel y el tajamar, como se muestra en la Figura 1.

Integra, junto con la Manzana Jesuítica en la ciudad de Córdoba y las Estancias de Caroya (1616), Santa Catalina (1622), Alta Gracia (1643) y La Candelaria (1683), el Camino Real de las Estancias Jesuíticas. Reconociendo sus valores patrimoniales únicos asociados a los testimonios jesuíticos en Córdoba, la UNESCO los ha inscripto en la Lista de Patrimonio de la Humanidad en el año 2000.

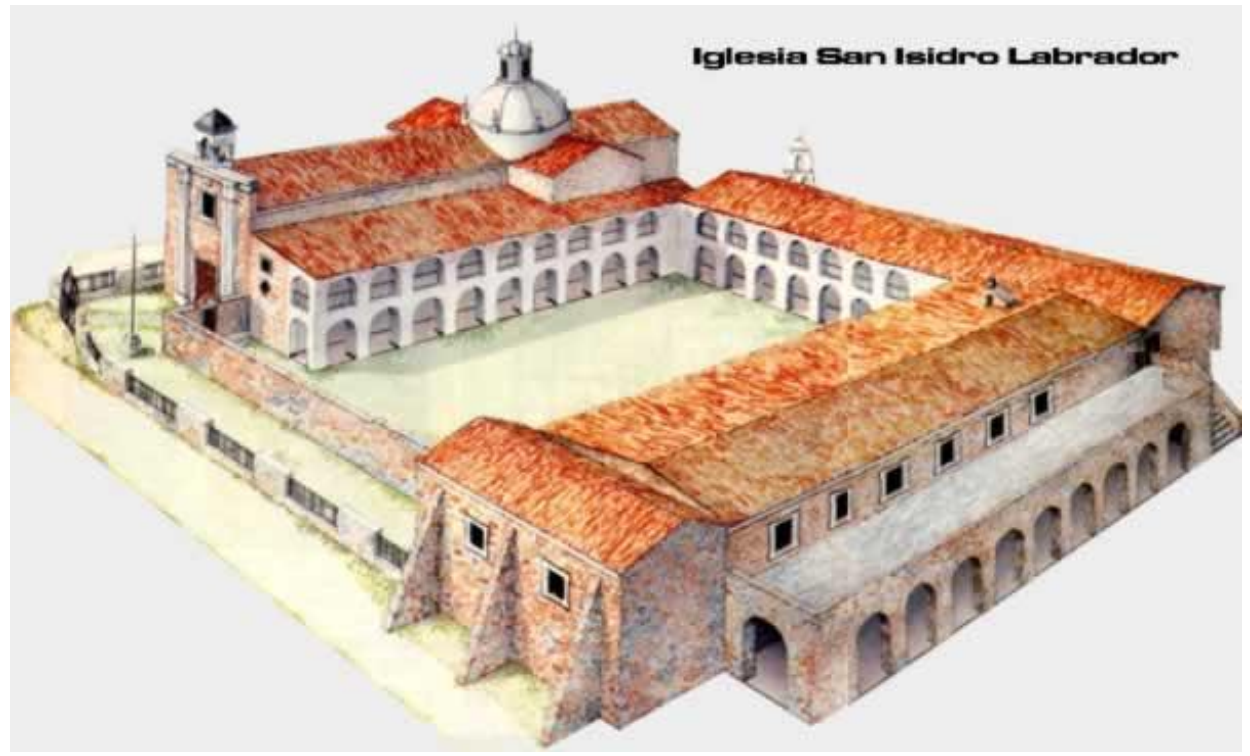

Figura 1. Vista del conjunto

Tras una semana de intensas lluvias, el 8 de mayo de 2018, se derrumbó el techo de la Cocina Histórica de la Estancia de Jesús María, Museo Jesuítico, dejando inhabilitada parte del monumento que tiene varias zonas vedadas: cocina, fregadero, cúpula y medio parque, como se muestra en las Figura 2.

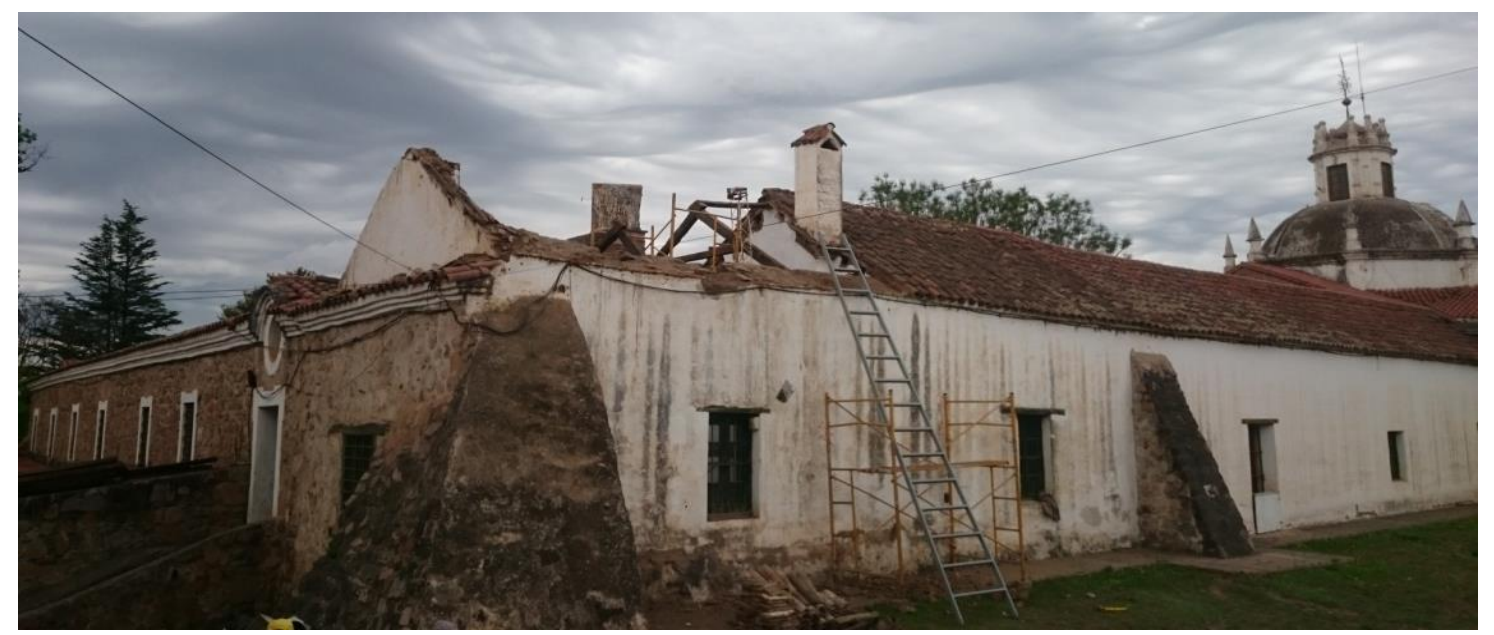

Figura 2. Vista del sector afectado por el derrumbe de la cubierta 


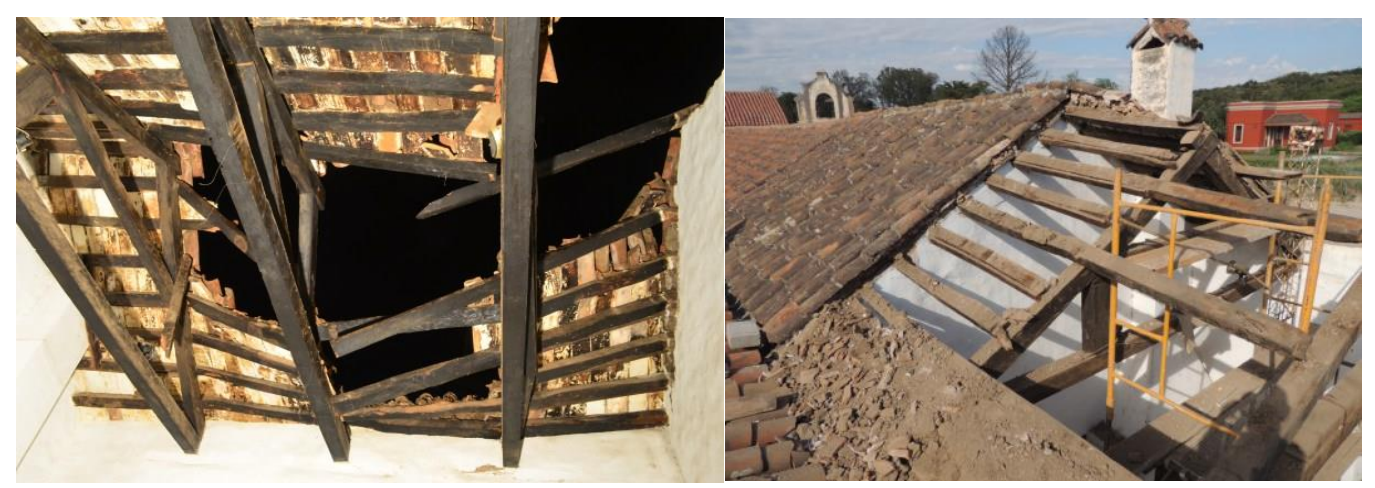

Figura 3. Vista interior de la estructura colapsada y remoción de las piezas estructurales

Se realizaron tareas de remoción de las tejuelas, tejas, bovedilla y tirantes de madera que se retiraron de la "cocina" de la Estancia, donde se produjo el derrumbe, ver Figuras 3 y 4.

En este contexto, a pedido de las autoridades del Museo, se analizan las posibles causas que han producido el derrumbe parcial del techo de una sala de la Estancia de Jesús María - Museo Jesuítico Nacional, ocurrido en el año 2018.

El objetivo es elaborar recomendaciones y una propuesta de recuperación de la estructura para incorporar a un proyecto de restauración integral que respete la autenticidad del edificio y la conservación de los elementos constitutivos, sabiendo su origen, sus técnicas, y la forma en que fue diseñada y construida una de las techumbres originales del conjunto que construyeron los jesuitas durante el siglo XVIII.

\section{ANTECEDENTES}

El presente trabajo tiene como antecedente el estudio y verificación realizados sobre la misma construcción durante el año 2008 por un equipo de investigación del TIDE, FAUD, UNC, en oportunidad de realizarse un trabajo de investigación en el Museo Jesuítico Nacional de Jesús María.

\subsection{Análisis y Verificación de la Cabriada en Cocina de Planta Alta}

La estructura que se verificó en esa oportunidad corresponde al plano superior de la sala ubicada en planta alta cuyo destino original era la cocina y que data de la construcción del museo no habiéndose realizado intervención alguna en dicha estructura, ver Figura 2.

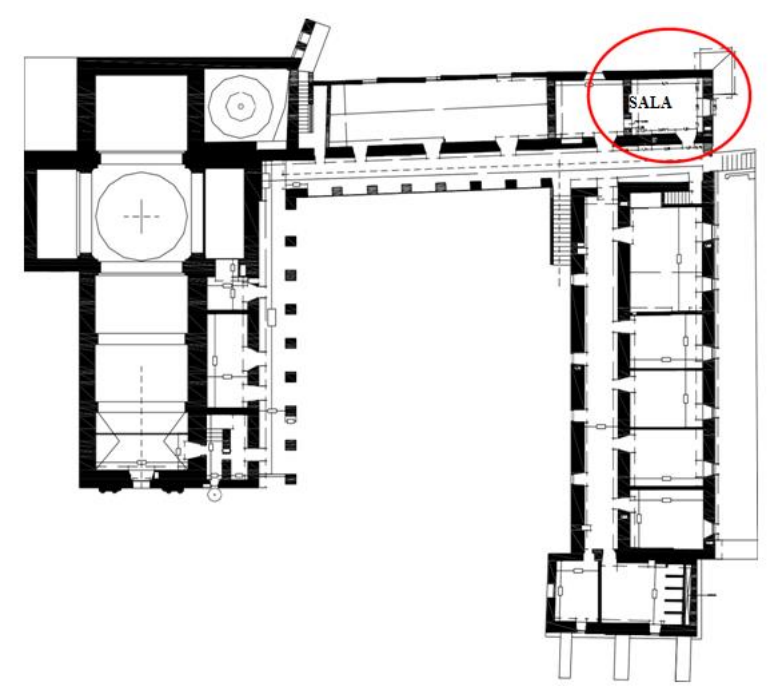

Figura 2. Planta Alta del Museo y ubicación de la Sala 


\subsubsection{Relevamiento de la estructura}

Como primera medida se procedió a relevar in situ la sala en cuestión, tanto en sus dimensiones en planta, como así también la organización estructural del plano superior. Para el relevamiento en alzado se utilizaron además los datos suministrados por el equipo de topógrafos.

La estructura del techo de la sala está conformado por tres vigas reticuladas de madera azuelada conformadas por pares, nudillo y tensor, conformación ésta característica de la época, como muestra la Figuras 3, 4 y 5.

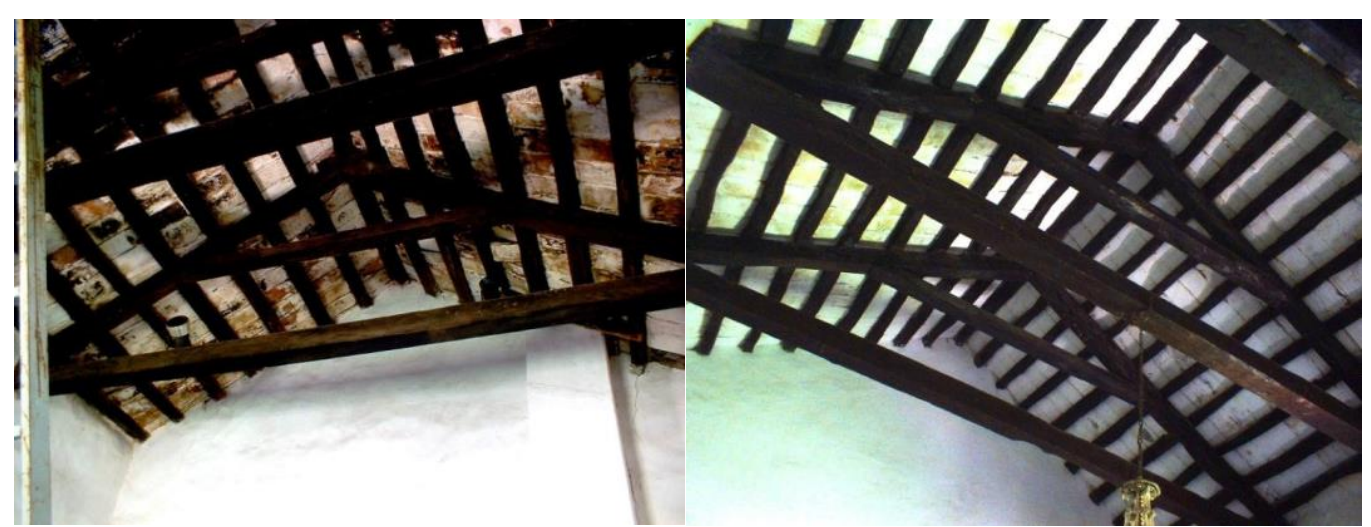

Figura 3. Cabriada de cocina

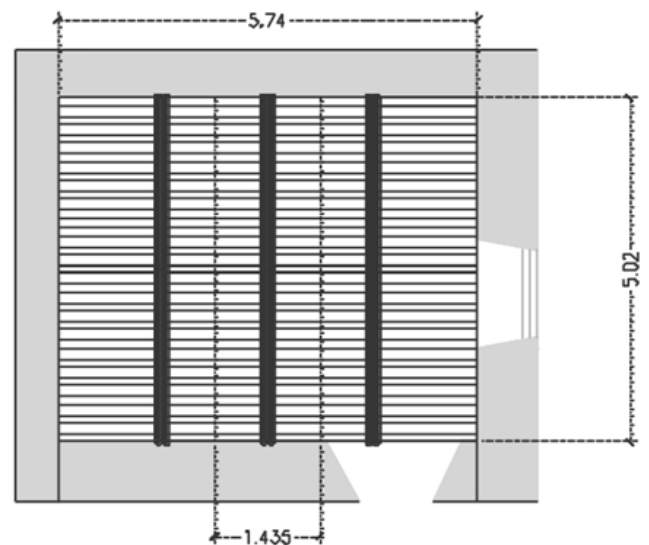

Separación entre ejes de cabriadas

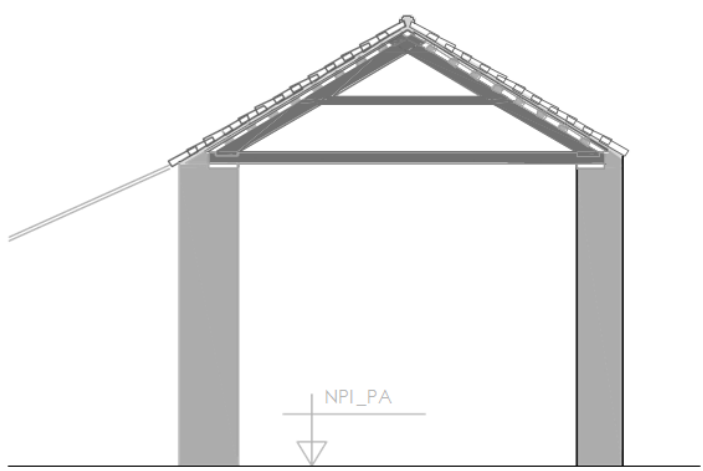

CORTE TRANSVERSAL DE LA SALA

Figura 4. Organización Estructural del Techo de la Sala. Corte Transversal

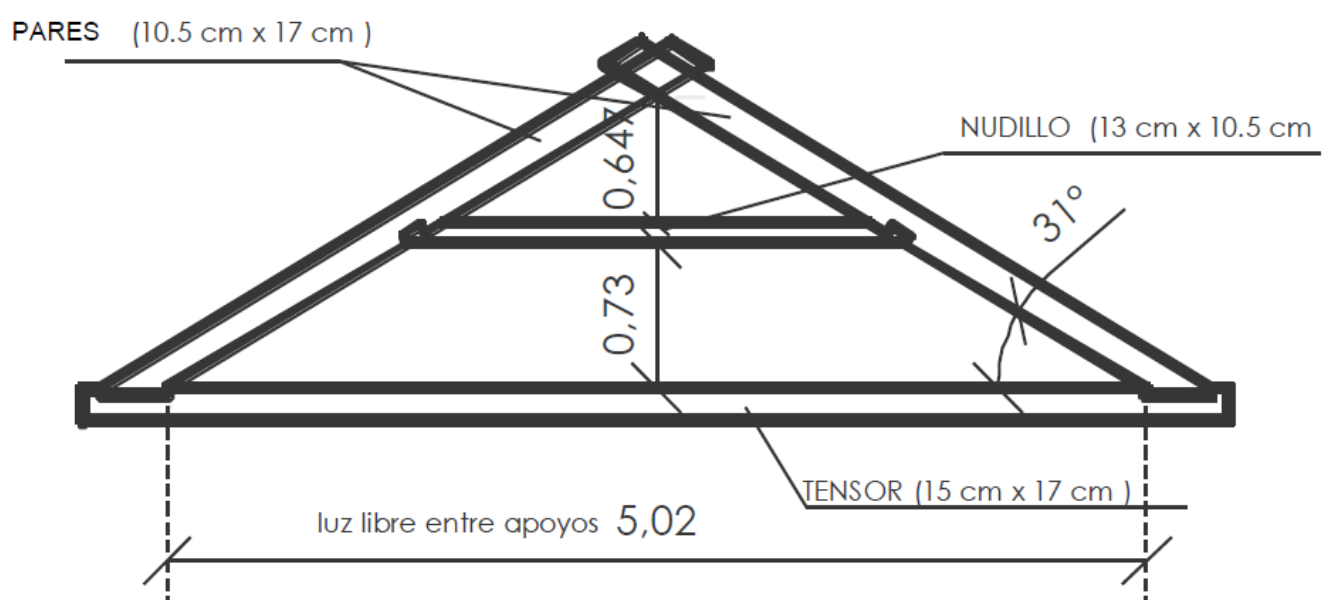

Figura 5. Dimensiones viga, par, nudillo y tensor 


\subsubsection{Características de la Madera de la cabriada}

Se realizó el estudio macro y microscópico de una muestra enviada para la identificación de la madera (peritaje Técnico en Anexo, elaborado por el Laboratorio de Dendrología y Xilología, INSIMA, Facultad de Ciencia Forestales de la Universidad Nacional de Santiago del Estero) obteniéndose los siguientes resultados:

Especie: Prosopis sp. (ALGARROBO) con presencia de galería de insectos, como se puede apreciar en las Figuras 6 y 7.
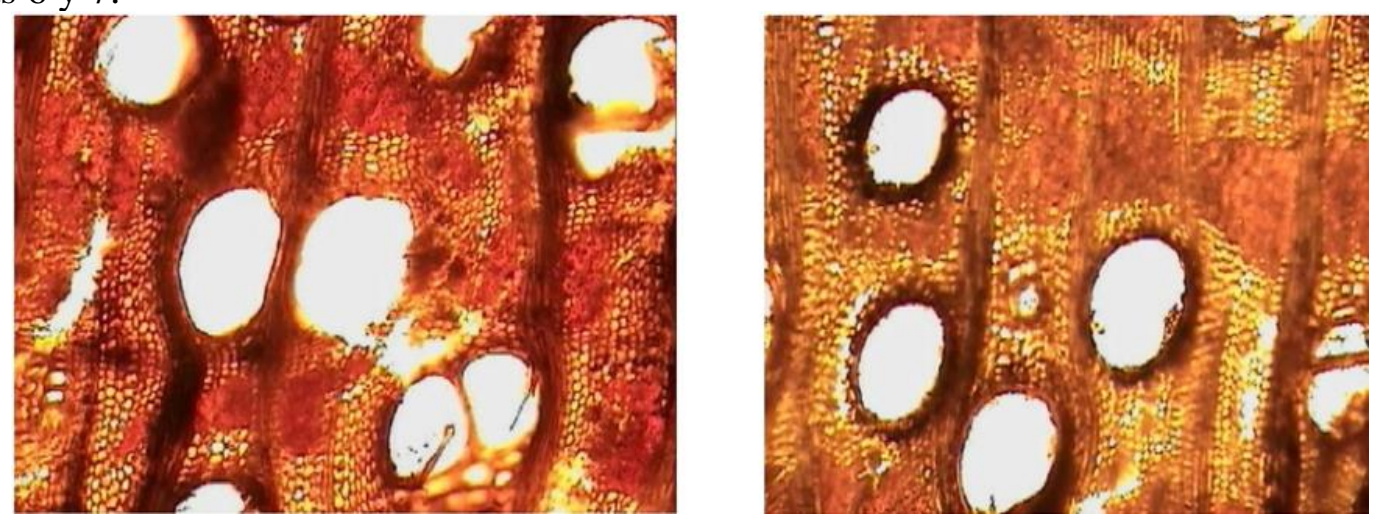

Figura 6. Muestra con presencia de galerías de insectos

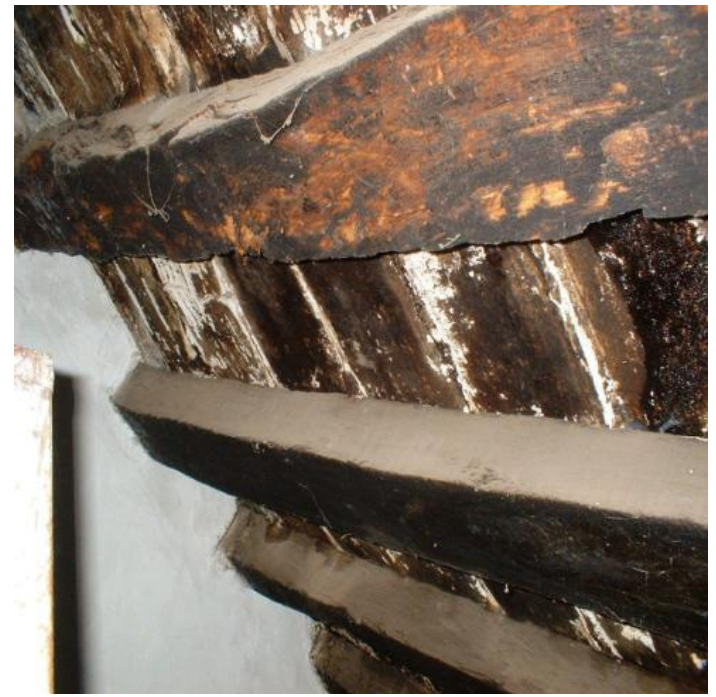

Figura 7. Estado de la madera de la estructura

Se realizaron ensayos en el Laboratorio del Taller de Investigación y Diseño Estructural, de la Facultad de Arquitectura de la Universidad Nacional de Córdoba para conocer las características físicomecánicas de la madera, según Norma NBR 7190 (1)-(2): (Probeta libre de defectos)

Densidad $=690 \mathrm{~kg} / \mathrm{m}^{3}$

Módulo de Elasticidad Medio Em = $63667 \mathrm{~kg} / \mathrm{cm}^{2}$

Módulo de Elasticidad Efectivo (para verificación de la deformación teniendo en cuenta la fluencia lenta) (1):

Eef $=\operatorname{kmod} 1 \times \operatorname{kmod} 2 \times \operatorname{kmod} 3 \times \mathrm{Em}=24448.128 \mathrm{~kg} / \mathrm{cm}^{2}$

Resistencia característica: $\mathrm{fc} 0 \mathrm{k}=379 \mathrm{~kg} / \mathrm{cm}^{2}$

Resistencia de cálculo: fc0d $=\operatorname{kmod} 1 \times \operatorname{kmod} 2 \times \operatorname{kmod} 3 \times \mathrm{fc} 0 \mathrm{k} / 1,4=103.95 \mathrm{~kg} / \mathrm{cm}^{2}(2)$ 


\subsubsection{Determinación de las características de la Cubierta de techo sobre la cabriada:}

Se realizaron exploraciones en la cubierta para determinar los materiales con que estaba realizada, como así también los espesores de los mismos y poder cuantificar la carga por metro cuadrado de la misma, ver Figura 8.

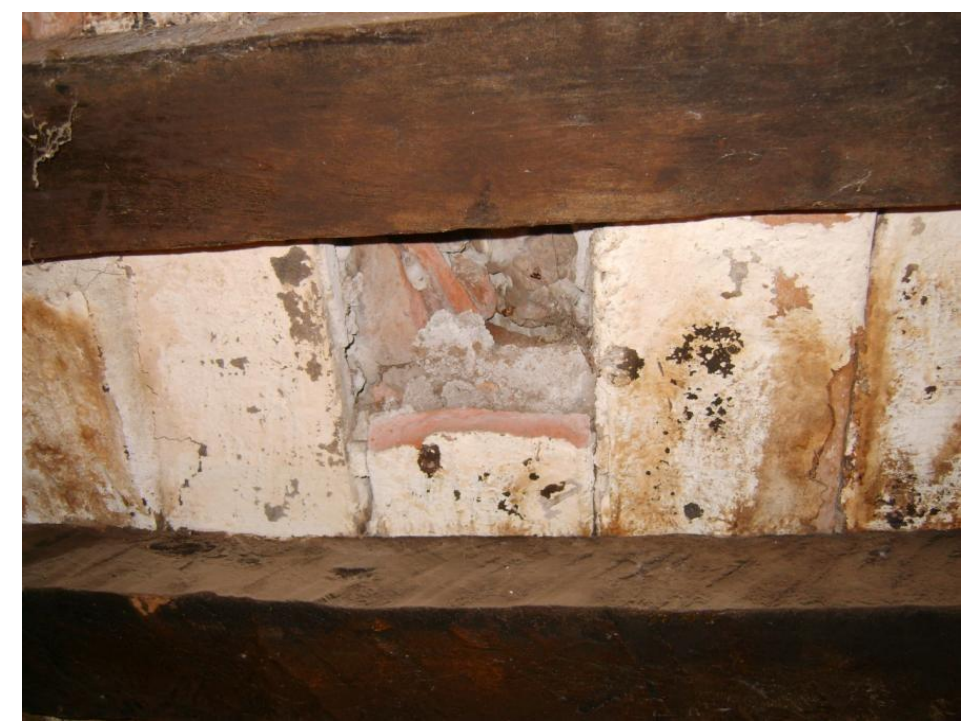

Figura 8. Composición y estado de la cubierta

Se determinó que la cubierta está conformada por bovedillas de $18 \mathrm{~cm} \mathrm{x} 28 \mathrm{~cm}$ x $3,6 \mathrm{~cm}$ de alto, asentadas sobre las correas, sobre las que se encuentran colocadas tejas españolas con una argamasa de $4 \mathrm{~cm}$ de espesor utilizada como mortero de asiento, como se muestra en la Figura 9.
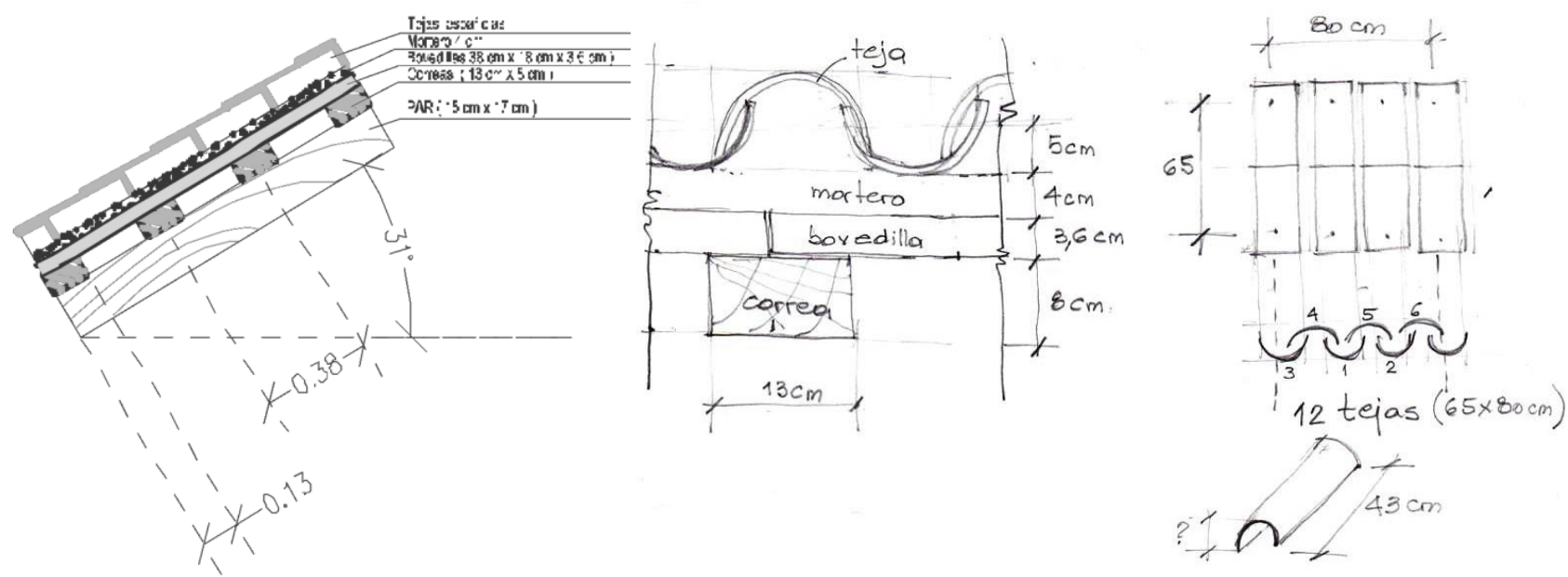

Figura 9. Corte longitudinal, corte transversal y detalles de la cubierta

\subsubsection{Análisis de cargas sobre la cabriada, cálculo de las solicitaciones y de la deformabilidad}

Una vez estudiada la conformación y geometría de la cubierta, se realiza el análisis de cargas sobre la misma.

Combinaciones de cargas (según lo establecen las Normas Brasileras BR7190):

- Para cálculo de las solicitaciones y verificación de la resistencia (3): 1,3 g + 1,4 p

$$
1,3 \times(0,4+0,012)+1,4 \times 0,025 \_\mathbf{q} \mathbf{1}=\mathbf{0 , 5 7 0} \mathbf{t} / \mathbf{m}(3)
$$


- Para verificar la deformabilidad (4): $1 \mathrm{~g}+0,2 \mathrm{p}$

$1 \times(0,4+0,012)+0,2 \times 0,025 \_\mathbf{q} 2=\mathbf{0 , 4 1 7} \mathbf{t} / \mathbf{m}(4)$

Para el cálculo de las solicitaciones y de la deformabilidad de la estructura, con los datos ya obtenidos se utilizó el software Strap, ver Figura 10 y Tabla 1.

\begin{tabular}{|c|c|c|}
\hline $\begin{array}{c}\mathrm{N}^{\circ} \mathrm{DE} \\
\text { BARRA }\end{array}$ & $\mathrm{b}(\mathrm{cm})$ & $\mathrm{h}(\mathrm{cm})$ \\
\hline $\mathbf{1}$ (Mamp) & 99 & 60 \\
\hline $\mathbf{2}$ & 10.5 & 17 \\
\hline $\mathbf{3}$ & 10.5 & 17 \\
\hline $\mathbf{4}$ & 10.5 & 17 \\
\hline $\mathbf{5}$ & 10.5 & 17 \\
\hline $\mathbf{6}$ (Mamp) & 70 & 60 \\
\hline $\mathbf{7}$ & 15 & 17 \\
\hline $\mathbf{8}$ & 13 & 10.5 \\
\hline
\end{tabular}

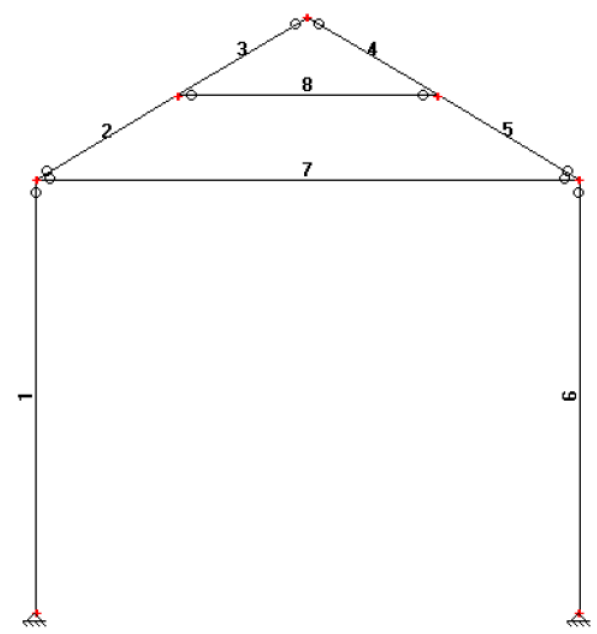

Figura 10. Modelo Estructural

Tabla 1. Datos materiales

\begin{tabular}{|c|c|c|c|c|c|c|}
\hline \multirow{2}{*}{ MATERIAL } & PESO ESPECÍFICO & \multicolumn{2}{c|}{ Em } & \multicolumn{2}{c|}{ Eef } \\
\cline { 2 - 7 } & $\mathbf{K g} / \mathbf{m}^{\mathbf{3}}$ & $\mathbf{t} / \mathbf{m}^{\mathbf{3}}$ & $\mathbf{K g} / \mathbf{c m}^{\mathbf{2}}$ & $\mathbf{t} / \mathbf{m}^{\mathbf{2}}$ & $\mathbf{K g} / \mathbf{c m}^{\mathbf{2}}$ & $\mathbf{t} / \mathbf{m}^{\mathbf{2}}$ \\
\hline Mampostería & 1300 & 1.3 & 16000 & 160000 & --------- & ---------- \\
\hline Algarrobo & 690 & 0.69 & 63667 & 636670 & 24448.1 & 244481 \\
\hline \multicolumn{3}{|c|}{} & \multicolumn{3}{|c|}{$(\mathrm{Kmod}=0.6 \times 0.8 \times 0.8)$} \\
\hline
\end{tabular}

En esa verificación se consideraron las dimensiones externas de los elementos de madera como si las secciones fueran macizas, siguiendo los lineamientos de la Norma Brasilera NBR 7190 basada en ensayos de probetas sin defecto de la especie identificada. Ver Figura 11 y Tabla 2.
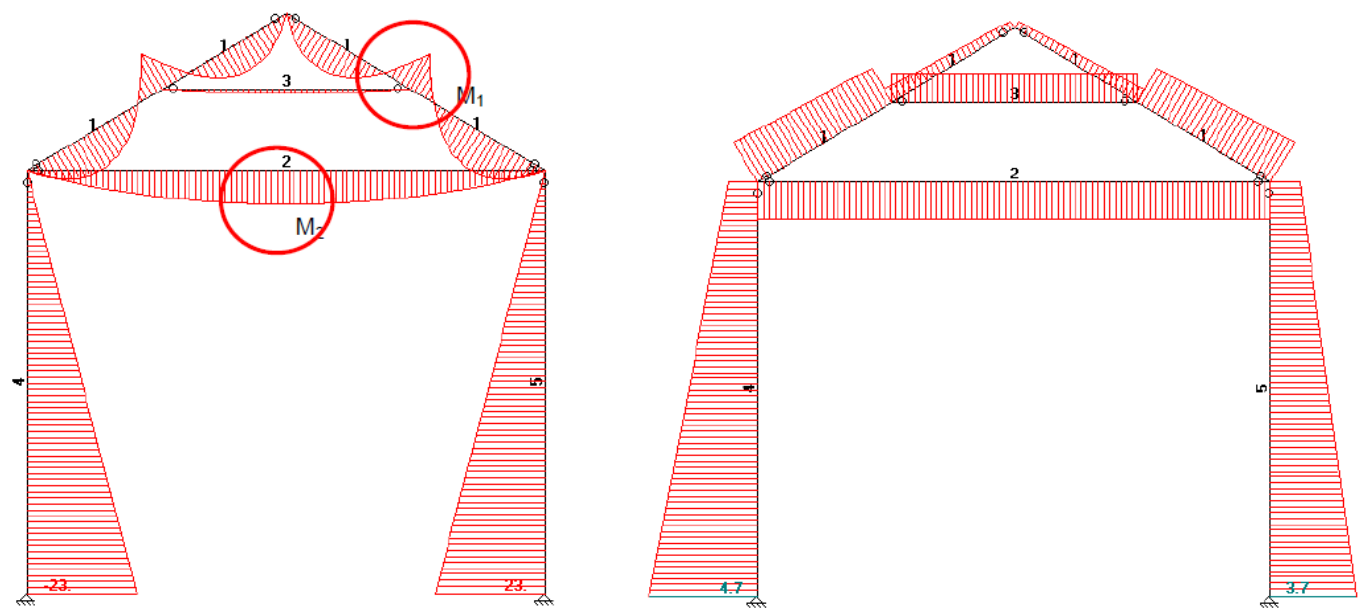

Figura 11. Diagrama de Momentos Flectores y Esfuerzos Normales 
Tabla 2. Máximas solicitaciones en barras

\begin{tabular}{|c|c|c|c|}
\hline $\mathbf{N}^{\mathbf{0}}$ de barra & Denominación & $\begin{array}{c}\text { Esfuerzo de } \\
\text { Tracción }\end{array}$ & $\begin{array}{c}\text { Esfuerzo de } \\
\text { Compresión }\end{array}$ \\
\hline 7 & tensor & $3204 \mathrm{~kg}$ & ----------- \\
\hline 8 & nudillo & ----------- & $1321 \mathrm{Kg}$ \\
\hline $2,3,4,5$ & pares & ----------- & $3738 \mathrm{~kg}$ \\
\hline
\end{tabular}

- Verificación seccional de los pares (5):

$\mathrm{M} 1=10.91 \mathrm{Tcm} ; \mathrm{N}=2.67 \mathrm{t}$

$\sigma=51,08 \mathrm{~kg} / \mathrm{cm}^{2} \leq 103.95 \mathrm{~kg} / \mathrm{cm}^{2} \operatorname{VERIFICA}(5)$

- Verificación seccional del tensor (6):

$\sigma=22,38 \mathrm{~kg} / \mathrm{cm}^{2} \leq 103.95 \mathrm{~kg} / \mathrm{cm}^{2} \operatorname{VERIFICA}(6)$

- Verificación de la deformabilidad:

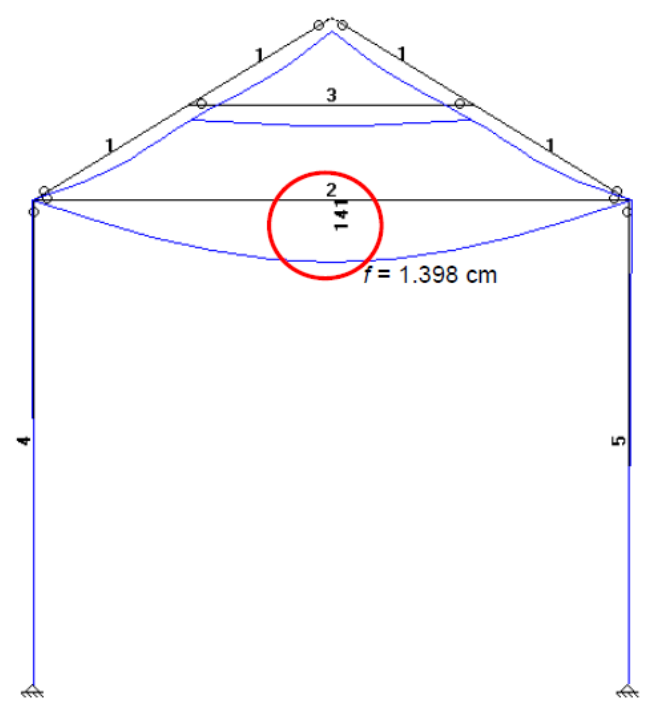

Figura 12. Descensos Máximos

Dada la irregularidad del tensor la medición realizada en obra del descenso arrojó un valor aproximado de $1 \mathrm{~cm}$.

Flecha Máxima de Cálculo $=1.398 \mathrm{~cm}>$ Flecha Máxima relevada $=1 \mathrm{~cm}$.

En cuanto máximo descenso de la viga reticulada, menor que el esperado, y contrariamente a lo que ocurre cuando las uniones son mecánicas (bulones), en este caso las uniones tradicionales por encastre no dan lugar a una mayor deformación.

Con respecto a las galerías de insectos, que debilitan la madera y facilitan su putrefacción, se recomendó en primer lugar realizar reparaciones necesarias para impedir el aumento de contenido de humedad de la madera, aplicar insecticidas líquidos en los muros en lugares donde estén en contacto con las vigas, y luego realizar el tratamiento de la madera original utilizando un insecticida fungicida específico. 


\section{ANALISIS Y VERIFICACION PARA LA SITUACION ACTUAL}

Ocurrido el derrumbe, se retiraron y clasificaron las diferentes piezas constitutivas de la cubierta: tejuelas, tejas, bovedilla y barras de madera, como se muestra en la Figura 13.

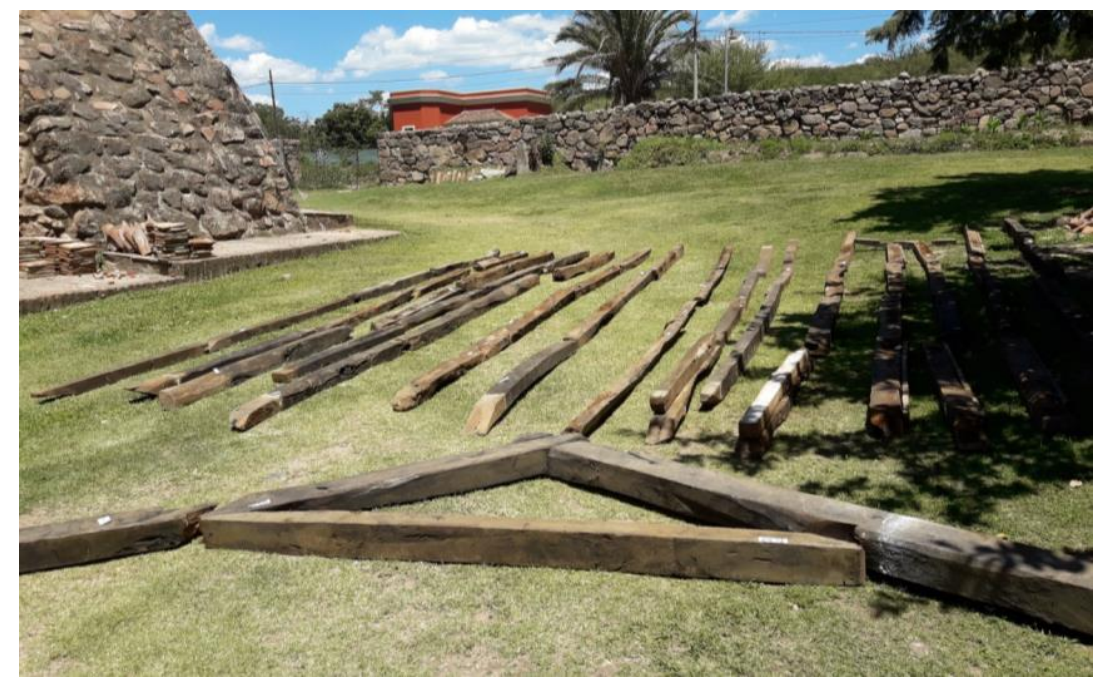

Figura 13. Piezas de madera clasificadas

\subsection{Ensayos y verificaciones realizadas}

Estos ensayos se realizaron en el Laboratorio del Taller de Investigación de Diseño Estructural (TIDE) de la Facultad de Arquitectura de la Universidad Nacional de Córdoba.

Se extrajo una muestra del par de la cabriada afectada, obteniendo una probeta de 5 x 5 x $15 \mathrm{~cm}$, como prescribe la norma NBR 7190, para realizar ensayo a compresión; Ver Figuras 14 y 15, y Tabla 3.

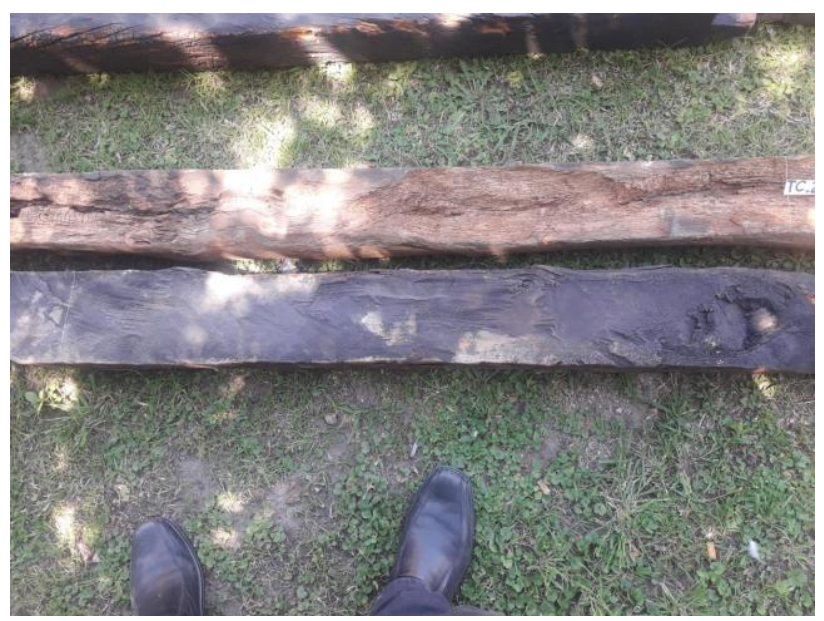

Figura 14. Piezas de madera para ensayar. Par y correa.

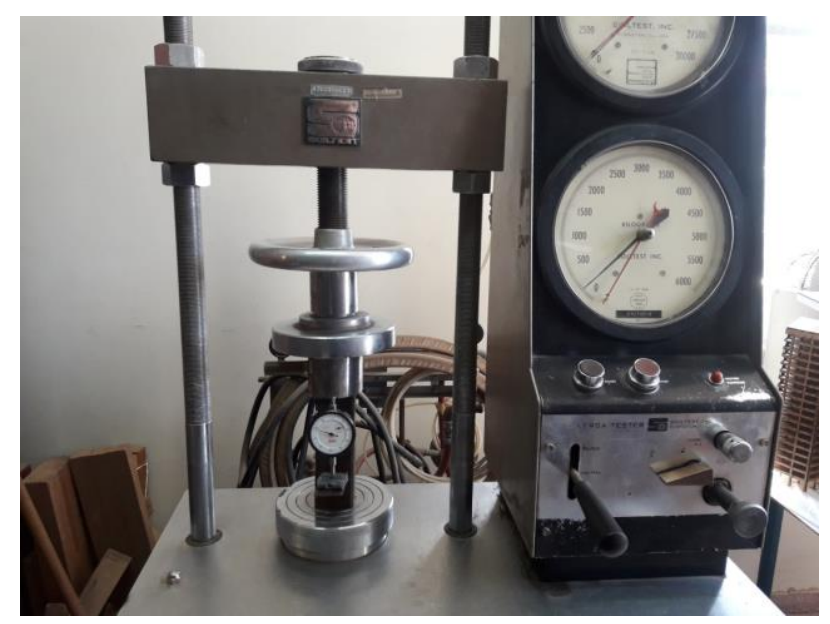

Figura 15. Ensayo a compresión de probeta extraída de un par de la cabriada 
Tabla 3. Planilla de ensayo a compresión paralelo a las fibras

MUESTRA DE PAR DE SALA 25 - COCINA JESUÍTICA EN ESTANCIA DE JESUS MARIA Ensayo de compresión paralelo a las fibras - Figura 15

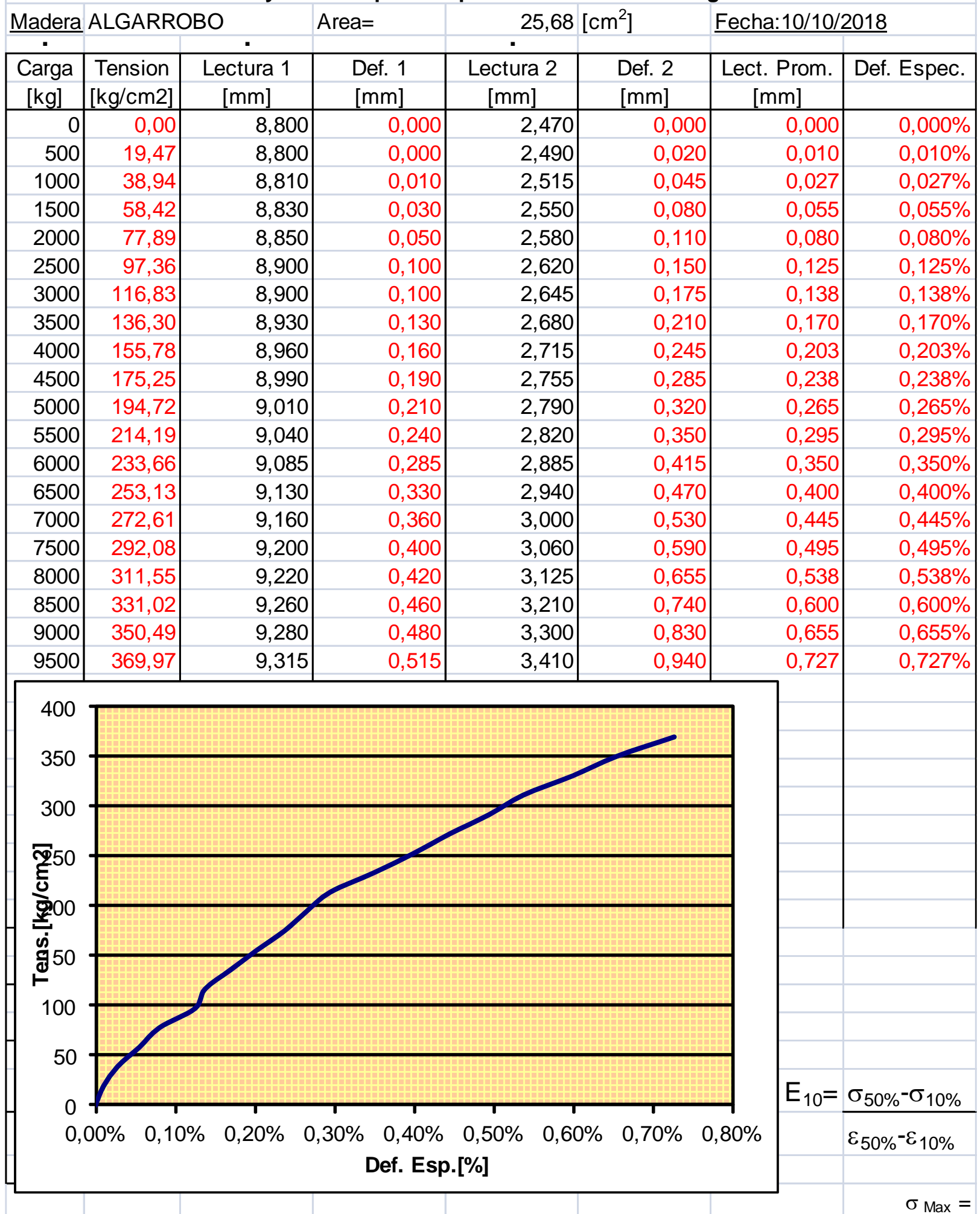

Por haber realizado un solo ensayo para no destruir más el elemento original, consideramos como tensión característica de esta madera (7) 
$\mathrm{fcok}=0.7 \times 369,97=259 \mathrm{Kg} / \mathrm{cm}^{2} .(7)$

Por lo tanto la resistencia de diseño (8):

$\mathrm{fcod}=\operatorname{Kmod} \times \mathrm{fc} 0 \mathrm{k} / 1,4=0,384 \times 259 / 1,4=71 \mathrm{Kg} / \mathrm{cm}^{2}(8)$

En los análisis registrados en los antecedentes la tensión de trabajo en la sección más solicitada del par es de $51,08 \mathrm{Kg} / \mathrm{cm}^{2}$ menor que $71 \mathrm{Kg} / \mathrm{cm}^{2}$

Del resultado de este ensayo, sería aconsejable tratar de recuperar la estructura utilizando los elementos que tenía, corrigiendo con técnicas adecuadas la disminución y/ó roturas seccionales de los mismos.

También se pudo ensayar a flexión una correa perteneciente al mismo techo, como se ve en la Figura 16.

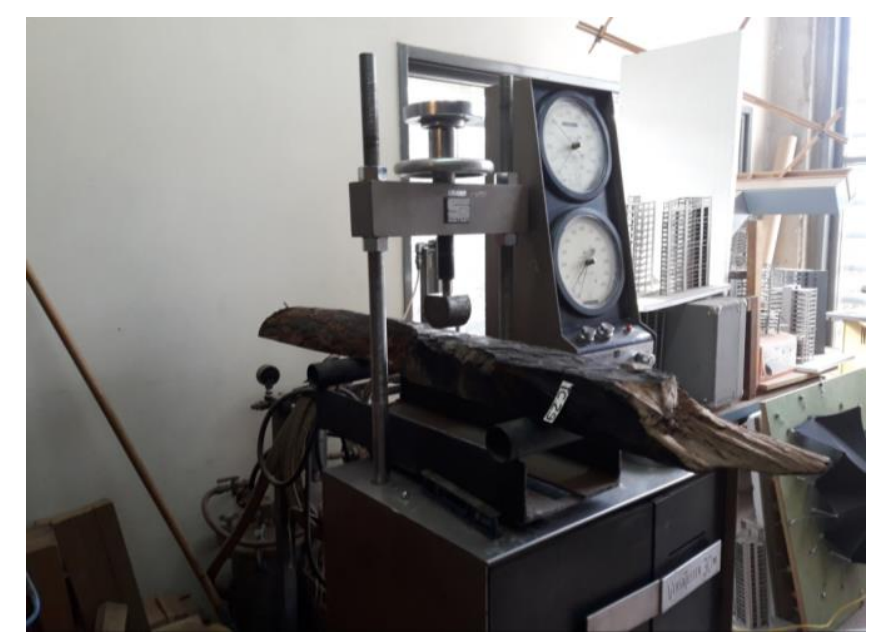

Figura 16. Ensayo a flexión de una correa de cubierta

Se evaluaron dos secciones, en virtud de la irregularidad de la sección y pérdida de material por deterioro, para verificar la capacidad resistente. Ver las Tablas 4 y 5.

Tabla 4. Verificación correa Sección 1

\begin{tabular}{|c|c|c|}
\hline SECCIÓN $(\mathrm{cm})$ & AREA $(\mathrm{cm} 2)$ & Sx $(\mathrm{cm} 3)$ \\
\hline & & \\
\hline & 123,5 & 195,54 \\
\hline
\end{tabular}

\begin{tabular}{|r|c|c|c|}
\hline CARGA ( KG) & LECTURA $(\mathrm{mm})$ & $\mathrm{M}(\mathrm{Kgcm})$ & TENSIÓN $(\mathrm{Kg} / \mathrm{cm} 2)$ \\
\hline 0 & 5,20 & 0 & 0,0 \\
\hline 500 & 7,70 & 8125 & 41,6 \\
\hline 1000 & 9,20 & 16250 & 83,1 \\
\hline 1500 & 1,10 & 24375 & 124,7 \\
\hline 1600 & rotura & 26000 & 133,0 \\
\hline
\end{tabular}


Tabla 5. Verificación correa Sección 2

\begin{tabular}{|c|c|c|c|c|}
\hline SECCIÓN $(\mathrm{cm})$ & AREA $(\mathrm{cm} 2)$ & Ix $(\mathrm{cm} 4)$ & Sx1 $(\mathrm{cm} 3)$ & Sx2 $(\mathrm{cm} 3)$ \\
\hline & & & & \\
\hline
\end{tabular}

\begin{tabular}{|r|c|c|c|c|}
\hline CARGA (KG) & LECTURA $(\mathrm{mm})$ & $\mathrm{M}(\mathrm{Kgcm})$ & TENSIÓN T $(\mathrm{Kg} / \mathrm{cm} 2)$ & TENSIÓN C $(\mathrm{Kg} / \mathrm{cm} 2)$ \\
\hline 5 & 1,60 & 0 & 0,0 & 0,0 \\
\hline 500 & 3,40 & 8125 & 87,5 & 128,8 \\
\hline 750 & 4,10 & 12187,5 & 131,3 & 193,2 \\
\hline 1000 & 5,10 & 16250 & 175,1 & 257,6 \\
\hline
\end{tabular}

- Carga sobre cada correa (9):

Carga de cubierta $295,7 \mathrm{Kg} / \mathrm{m}^{2}$ (incluida sobrecarga de uso)

Carga por metro lineal de correa 295,7 x 0,38 $=112,37 \mathrm{Kg} / \mathrm{m}(9)$

Luz de cálculo para $1=1,44 \mathrm{~m}$

- Solicitación (10):

Momento flector máximo $\mathrm{M}=\mathrm{q} \times 1^{2} / 8=29,13 \mathrm{Kgm}(10)$

- Resultados Ensayos

Para los dos ensayos, el momento flector menor que produjo la rotura fue de $170 \mathrm{Kgm}$, mucho mayor que el máximo momento solicitante sobre la correa de 29,13 Kgm.

Aunque los resultados de estos ensayos indican una resistencia menor que las obtenidas con maderas sin defectos, vemos en el análisis anterior que se pueden reutilizar conservando la seguridad reglamentaria, por lo que se aconseja recuperar los elementos dañados utilizando la madera original, realizando refuerzos y uniones con varillas roscadas zincadas y resinas epoxi en cada situación, de acuerdo a bibliografía especializada en recuperación de estructuras de maderas en edificios patrimoniales.

\section{PROYECTO DE RECUPERACION DE ZONAS AFECTADAS EN DISTINTOS ELEMENTOS}

Cuando las zonas afectadas han sido producidas por pudriciones o a la acción de insectos y es superficial, está indicado el saneamiento de la parte afectada y la reconstrucción con resina o mortero epoxídico. Si es necesario, es posible el aumento de la altura del elemento, como se muestra en la Figura 17. La superficie de contacto se aumenta por ranurado y se mejora insertando conectores metálicos zincados. 


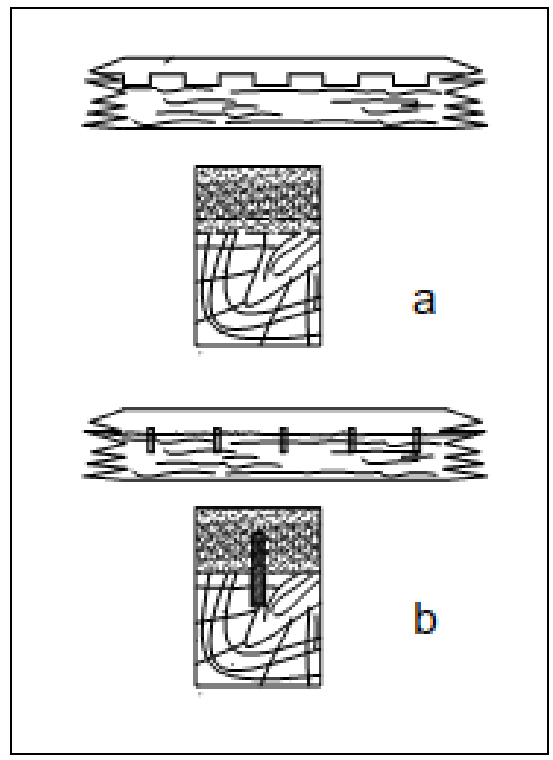

Figura 17. Reconstrucción de la sección.

Cuando la zona afectada coincide con los apoyos sobre los muros de cerramiento faltando sección en el comienzo del mismo o se ha producido la rotura en el tramo del elemento, se recurre a una sustitución parcial por prótesis enlazada a la madera sana a través de varillas de acero zincado, como se indica en la Figura 18. La prótesis puede reconstruirse con mortero epoxídico como indica la Figura 19.

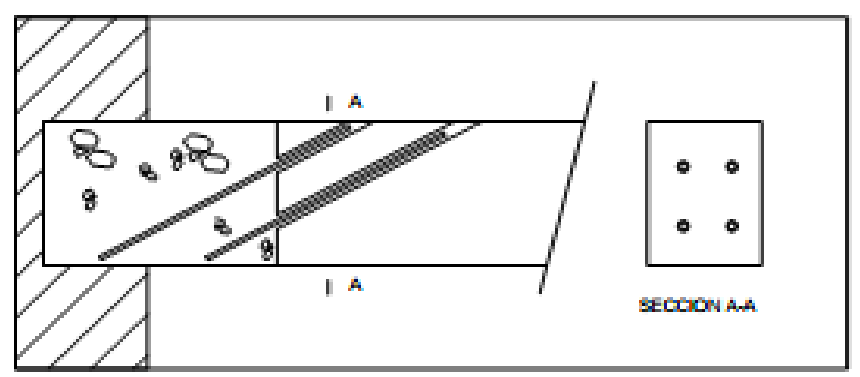

Figura 18. Sustitución parcial por prótesis enlazada a la madera sana a través de varillas de acero

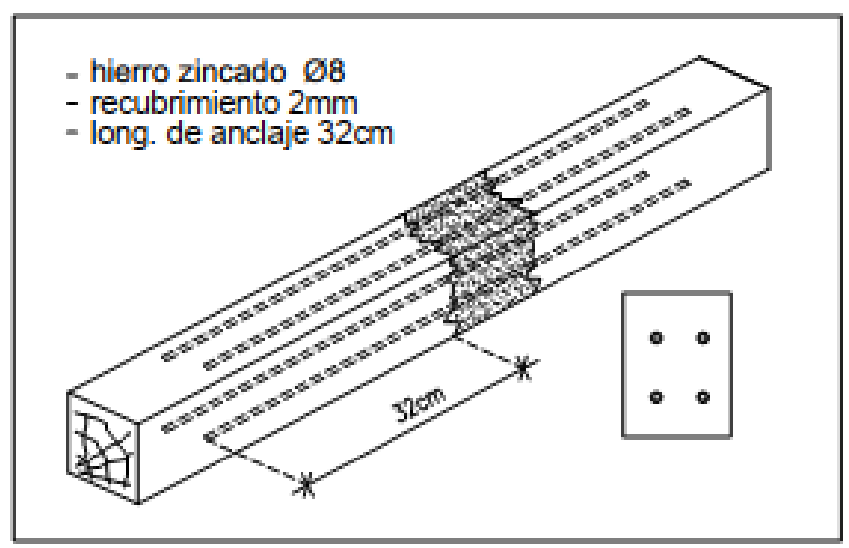

Figura 19. Reconstrucción de la sección.

Esta metodología ya se ha experimentado en nuestra zona en la recuperación de los arcos y vigas de la capilla doméstica de la residencia jesuítica y en la iglesia de Salsipuedes, Córdoba. 


\section{CONCLUSIONES}

Aunque los resultados de estos ensayos indican una resistencia menor que las obtenidas con maderas sin defectos, vemos en el análisis anterior que se pueden reutilizar conservando la seguridad reglamentaria.

Es por ello que se aconseja recuperar los elementos dañados utilizando la madera original, realizando refuerzos y uniones con varillas roscadas zincadas y resinas epoxi en cada situación, de acuerdo a bibliografía especializada en recuperación de estructuras de maderas en edificios patrimoniales.

\section{AGRADECIMIENTOS}

Personal del Museo Jesuítico Nacional de Jesús María.

\section{REFERENCIAS}

Argüelles Álvarez, R.; Arriaga Martitegui, F. (2000). "Estructuras de madera. Diseño y Cálculo" Asociación de Investigación Técnica de las Industrias de la Madera y el Corcho. España. Associação Brasileira de Normas Técnicas. (1997). NBR 7190: Projeto de Estruturas de Madeira. Río de Janeiro.

Gomez, J.L. (2000). "Estructuras de madera” Edit. Ingreso, Córdoba. Argentina

Gómez, J.L. et al. (2009-2010) Proyecto de investigación "Diseño de uniones de madera basado en la deformación de la estructura. Verificación y recuperación de obras de valor patrimonial"

Gómez, J.L. et al. (2010) "Durabilidad y Deformabilidad de Vigas de Madera Construidas en el Museo Jesuítico Nacional de Jesús María - Siglo XVII” CONPAT 2010.

Gómez, J.L. et al. (2010) "Fundamento la recuperación de la estructura de madera de la capilla domestica de la Residencia Jesuítica y la decisión de preservar una parte en estado original”. XX Jornadas Argentinas de Ingeniería Estructural.

Gómez, J.L. et al (2015) "Recuperación de un techo de madera con manifestaciones patológicas en su estructura" CONPAT 2015- Lisboa- Portugal

Lozano Apolo, G.; Lozano Martinez Luengas, A. (1996). "Técnicas de Intervención en el Patrimonio Arquitectónico" Catedráticos de la Universidad de Oviedo. Ed. Consultores Técnicos de Construcción, España 